\title{
Plástica da valva mitral: resultados aos 17 anos de experiência
}

\author{
Pablo M. A. POMERANTZEFF*, Carlos M. A. BRANDÃO*, Cristiano N. FABER ${ }^{*}$, Marcelo H. FONSECA**, \\ Luiz B. PUIG*, Max GRINBERG*, Luís F. CARDOSO*, Flávio TARASOUTCHI*, Noedir A. G. STOLF*, \\ Geraldo VERGINELLI* ${ }^{*}$ Adib D. JATENE*.
}

Pomerantzeff P M A, Brandão C M A, Faber C N, Fonseca M H, Puig L B, Grinberg M, Cardoso L F, Tarasoutchi F, Stolf N A G, Verginelli G, Jatene A D - Plástica da valva mitral: resultados aos 17 anos de experiência. Rev Bras Cir Cardiovasc 1999; 14 (3): 185-90.

RESUMO: Foram analisados 545 pacientes submetidos a 560 plásticas da valva mitral no período de março de 1980 a dezembro de 1997. A idade variou de 3 meses a 86 anos com média de idade de 42,2 $\pm 21,4$ anos. Duzentos e setenta e quatro $(50,3 \%)$ pacientes eram do sexo masculino. A etiologia foi reumática em $234(42,9 \%)$ pacientes. As técnicas utilizadas foram: ressecção quadrangular da cúspide posterior em 204 $(36,5 \%)$ pacientes, anuloplastia com tira posterior de pericárdio bovino em $139(24,5 \%)$ pacientes, anel de Carpentier em 102 (18,2\%) pacientes, anuloplastia posterior segmentar em 37 (6,6\%) pacientes e outras técnicas utilizadas em menor freqüência. Técnicas associadas foram empregadas em 199 (35,6\%) pacientes, sendo a mais freqüente o encurtamento de cordas em 72 (12,8\%). Operações associadas foram realizadas em 267 (47,8\%) pacientes. A mortalidade hospitalar foi de 3,7\% (21 pacientes). As taxas linearizadas dos eventos no pós-operatório foram $2,9 \%$ pacientes/ano para reoperação, $0,6 \%$ pacientes/ano para tromboembolismo, $0,3 \%$ pacientes/ano para endocardite e $0,1 \%$ pacientes/ano para hemólise. A sobrevida actuarial foi de 76,8 $\pm 10,8$ em 17 anos e sobrevida livre de endocardite, tromboembolismo, reoperação e hemólise no mesmo período foi respectivamente $98,9 \pm 0,6 \%, 93,9 \pm 3,7 \%, 61,0 \pm 7,9 \%$ e $99,7 \pm 0,2 \%$. Podemos concluir que os pacientes submetidos à plástica da valva mitral apresentaram evolução satisfatória.

DESCRITORES: Valva mitral, cirurgia. Doença das valvas cardíacas, cirurgia.

\section{INTRODUÇÃO}

As técnicas de plastia valvar são cada vez mais utilizadas no manuseio cirúrgico das lesões valvares e são preferíveis à troca valvar quando tecnicamente possíveis. Alguns obstáculos à utilização das diversas técnicas existentes incluem a incerteza do cirurgião quanto ao sucesso da reconstrução, bem como em relação à durabilidade do procedimento a longo prazo (1).

O sucesso da operação conservadora depende de um profundo conhecimento da estrutura dinâmica tridimensional da valva e do seu funcionamento mecânico normal através do ciclo cardíaco. Neste sentido o ecoDopplercardiograma transtorácico é indispensável na análise pré-operatória para o planejamento da tática cirúrgica, e a utilização de transdutores transesofágicos é de grande valia para a análise intra-operatória da reconstrução (2).

Cabe ressaltar, que a disfunção valvar freqüentemente é resultado de diversas lesões afetando suas várias estruturas ${ }^{(3)}$. Portanto, é de extrema

Trabalho realizado no Instituto do Coração do Hospital das Clínicas da Faculdade de Medicina da Universidade de São Paulo, SP, Brasil. Apresentado ao $26^{\circ}$ Congresso Nacional de Cirurgia Cardíaca. Fortaleza, CE, 8 a 10 de abril, 1999.

* Do Instituto do Coração do Hospital das Clínicas da Faculdade de Medicina da Universidade de São Paulo

** Da Faculdade de Medicina da Universidade de São Paulo

Endereço para correspondência: Pablo M. A. Pomerantzeff. Av. Dr. Enéas de Carvalho Aguiar, 44. Divisão Cirúrgica. São Paulo, SP, Brasil. CEP: 05403-000.e-mail.: dclpablo@incor.usp.br 
Pomerantzeff P M A, Brandão C M A, Faber C N, Fonseca M H, Puig L B, Grinberg M, Cardoso L F, Tarasoutchi F, Stolf N A G, Verginelli G, Jatene A D - Plástica da valva mitral: resultados aos 17 anos de experiência. $\quad$ Rev Bras Cir Cardiovasc 1999; 14 (3): 185-90.

importância, durante o ato operatório, uma boa visibilização de todas as estruturas que compõem o aparelho valvar $(1,3)$ e após a correção cirúrgica é necessário que a área valvar esteja adequada e que haja uma boa área de coaptação entre as cúspides, o que certamente aumentará a durabilidade do procedimento (4).

Este estudo tem como objetivo relatar os resultados da plastia da valva mitral após 17 anos utilizando diversas técnicas de reconstrução.

\section{CASUÍSTICA E MÉTODOS}

No período entre março de 1980 e dezembro de 1997, 545 pacientes foram submetidos à plástica da valva mitral; a idade variou de 3 meses a 86 anos, com média de 42,2 $\pm 21,4$ anos. Duzentos e setenta e quatro $(50,3 \%)$ pacientes eram do sexo masculino e $271(49,7 \%)$ pacientes do sexo feminino.Em 408 $(74,8 \%)$ pacientes o diagnóstico foi de insuficiência mitral pura e $137(25,1 \%)$ eram portadores de dupla lesão mitral. A etiologia reumática em 234 (42,9\%) pacientes (Tabela 1). Como diagnósticos associados, $95(17,4 \%)$ pacientes apresentavam insuficiência tricúspide, 73 (13,9\%) insuficiência aórtica, 70 $(12,8 \%)$ insuficiência coronária, 31 (5,6\%) dupla lesão aórtica, 16 (2,9\%) endomiocardiofibrose, 13 (2,3\%) estenose aórtica, 9 (1,6\%) comunicação interatrial, $2(0,3 \%)$ comunicação interventricular, $1(0,1 \%)$ aneurisma de ventrículo esquerdo, 1 (0,1\%) aneurisma de aorta ascendente e $1(0,1 \%)$ paciente apresentava estenose tricúspide.

Foram utilizadas as seguintes técnicas cirúrgicas: ressecção quadrangular da cúspide posterior com plicatura do anel correspondente e sutura borda a borda das cúspides em 204 (36,5\%) pacientes, tira posterior de pericárdio bovino em 139 (24,8\%), anuloplastia com anel de Carpentier em 102 (18,2\%), tira posterior segmentar em $37(6,6 \%)$, anuloplastia de Kay em 15 (2,7\%), anuloplastia posterior tipo

TABELA 1

ETIOLOGIA

\begin{tabular}{lcr}
\hline & $\boldsymbol{N}^{\circ}$ PACIENTES & $\%$ \\
\hline Febre Reumática & 234 & 42,9 \\
Degeneração Mixomatosa & 158 & 28,9 \\
Indefinida & 56 & 10,2 \\
Isquêmica & 35 & 6,4 \\
Congênita & 26 & 4,7 \\
Endomiocardiofibrose & 16 & 2,9 \\
Endocardite & 8 & 1,4 \\
Valvulite Crônica Calcificada & 8 & 1,4 \\
Miocardiopatia Dilatada & 4 & 0,7 \\
\hline
\end{tabular}

Shore ${ }^{(5)}$ em $11(1,9 \%)$, sutura de fenda em $9(1,6 \%)$, ressecção de cúspide anterior em 8 (1,4\%), anuloplastia posterior através de pontos tipo "duplo U" em oposição em 5 (0,8\%), anuloplastia de Reed em $4(0,7 \%)$, duplicação do orifício mitral em 3 $(0,5 \%)$, ressecção de cúspide posterior em $3(0,5 \%)$, anel de Puig-Massana em $2(0,3 \%)$ e ampliação da cúspide posterior em $2(0,3 \%)$ pacientes, totalizando 560 plásticas da valva mitral.

Foram realizadas técnicas associadas aos procedimentos citados em 199 (35,6\%) pacientes, sendo comissurotomia mitral em $75(13,4 \%)$, encurtamento de cordas tendíneas em $72(12,8 \%)$, descalcificação da valva mitral em $36(6,4 \%)$, papilarotomias múltiplas em 34 (6\%), ressecção em cunha da cúspide anterior em $11(1,9 \%)$, desbastamento da cúspide posterior em 6 (1\%), encurtamento de músculos papilares em $5(0,8 \%)$, deslizamento associado à ressecção da cúspide posterior em $5(0,8 \%)$, reconstrução da cúspide posterior em $5(0,8 \%)$, plicatura da cúspide posterior em $4(0,7 \%)$, retirada de cordas curtas em $3(0,5 \%)$ e desbastamento de músculo papilar em $1(0,1 \%)$ paciente.

Em 267 (47,8\%) pacientes realizaram-se operações associadas à plastia da valva mitral com predomínio da plastia da valva tricúspide em 95 (17\%) pacientes (Tabela 2).

Para avaliarmos o refluxo após a plastia da valva mitral realizamos a injeção de solução fisiológica no ventrículo esquerdo, bem como testes com o coração batendo. Tem sido rotina nos últimos anos a utilização do ecoDopplercardiograma transesofágico para avaliação intra-operatória da reconstrução da valva mitral.

A análise dos resultados foi realizada através da construção de curvas actuariais pelo método de Kaplan-Meier, e as taxas linearizadas de risco foram definidas como o número total de eventos dividido pela soma do tempo de seguimento $(6,7)$.

TABELA 2

PROCEDIMENTOS CIRÚRGICOS ASSOCIADOS À PLÁSTICA DA VALVA MITRAL

\begin{tabular}{lcc}
\hline OPERAÇÕES ASSOCIADAS & N $^{\circ}$ DE PACIENTES & $\%$ \\
\hline Plástica da Valva Tricúspide & 95 & 16,9 \\
Substituição da Valva Aórtica & 79 & 14,1 \\
Revascularização do Miocárdio & 70 & 12,5 \\
Plástica da Valva Aórtica & 20 & 3,5 \\
Comissurotomia Aórtica & 16 & 2,8 \\
Atriosseptoplastia & 9 & 1,6 \\
Ressecção de Endomiocardiofibrose & 5 & 0,8 \\
Substituição da Aorta Ascendente & 1 & 0,1 \\
Aneurismectomia de Ventrículo Esquerdo & 1 & 0,1 \\
Comissurotomia Tricúspide & 1 & 0,1 \\
\hline
\end{tabular}


Pomerantzeff P M A, Brandão C M A, Faber C N, Fonseca M H, Puig L B, Grinberg M, Cardoso L F, Tarasoutchi F, Stolf N A G, Verginelli G, Jatene A D - Plástica da valva mitral: resultados aos 17 anos de experiência. $\quad$ Rev Bras Cir Cardiovasc 1999; 14 (3): 185-90.

\section{RESULTADOS}

A mortalidade hospitalar foi de $3,7 \%$ (21 pacientes), tendo como causas a síndrome de baixo débito em $10(1,8 \%)$ pacientes, falência de múltiplos órgãos e sistemas em 7 (1,2\%), arritmia em 2 (0,3\%) e acidente vascular cerebral em $2(0,3 \%)$

As complicações hospitalares mais freqüentes foram o baixo débito cardíaco temporário em 39 (7,1\%) pacientes, arritmia em 25 (4,5\%), insuficiência renal em $9(1,6 \%)$, acidente vascular cerebral em $6(1,1 \%)$, sepse em $5(0,9 \%)$, falência de múltiplos órgãos e sistemas em $4(0,7 \%)$, endocardite em $2(0,3 \%)$, infarto agudo do miocárdio em $2(0,3 \%)$ e infecção pulmonar em $1(0,1 \%)$. No período préoperatório 191 (35\%) pacientes estavam em classe funcional IV, 305 (55,9\%) em classe funcional III e $49(8,9 \%)$, estavam em classe funcional II. No pósoperatório tardio $439(80,5 \%)$ pacientes estavam em classe funcional I, $37(6,7 \%)$ em classe funcional II, $33(6 \%)$ em classe funcional III e $3(0,5 \%)$ pacientes em classe funcional IV. O tempo de seguimento foi de 27,984 meses/pacientes.

A mortalidade tardia nesta população foi de 12 $(2,2 \%)$ pacientes (Tabela 3$)$. Foram realizadas 15 replastias e 26 reoperações para substituição da valva mitral, com tempo médio entre a plastia e a replastia de 48,6 meses e entre a plastia e a substituição valvar de 30,7 meses. Em 2 casos foi realizada uma segunda replastia.

As taxas linearizadas dos eventos hemólise, tromboembolismo, reoperação e endocardite na evolução tardia estão discriminadas na Tabela 4.

O porcentual de sobrevida pela curva actuarial

TABELA 3

CAUSAS DE MORTALIDADE TARDIA

\begin{tabular}{lc}
\hline ETIOLOGIA & $\boldsymbol{N}^{\circ}$ PACIENTES \\
\hline Insuficiência Cardíaca Congestiva & 6 \\
Desconhecida & 4 \\
Septicemia & 1 \\
Infarto Agudo do Miocárdio & 1 \\
\hline
\end{tabular}

TABELA 4

TAXAS LINEARIZADAS DOS EVENTOS (EVOLUÇÃO TARDIA)

\begin{tabular}{lcr}
\hline EVENTO & PACIENTES/ANO & $\boldsymbol{N}^{\mathbf{P}}$ \\
\hline Reoperação & 2,9 & 41 \\
Tromboembolismo & 0,6 & 8 \\
Endocardite & 0,3 & 4 \\
Hemólise & 0,1 & 1 \\
\hline
\end{tabular}

foi de $76,8 \pm 10,8 \%$ em 17 anos (Gráfico 1). O porcentual de sobrevida livre do evento endocardite $98,9 \pm 0,6 \%$, (Gráfico 2), livre do evento tromboembolismo 93,9 $\pm 3,7 \%$ (Gráfico 3), livre do evento reoperação $61,0 \pm 7,9 \%$ (Gráfico 4 ) e livre do evento hemólise 99,7 \pm 0,2\% (Gráfico 5).

\section{COMENTÁRIOS}

Desde 1957, quando LILLEHEI et al. (8) relataram a primeira reconstrução valvar sob visão direta, um grande número de trabalhos tem sido publicado na literatura $(3-5,9,10)$. A doença degenerativa da valva mitral é a causa mais comum de regurgitação mitral nos países industrializados ${ }^{(9,10)}$. Nestes casos a plastia da valva mitral é procedimento de escolha, com excelentes resultados tardios (9-11). No entanto, em nosso meio a etiologia reumática é predominante e nesta população os resultados tardios podem ser prejudicados pelo aparecimento de novos surtos da doença (12). Apesar deste fato, todo esforço deve ser feito no sentido de se realizar o tratamento conservador uma vez que uma grande parte destes doentes é jovem e portanto sujeita a um número maior de intervenções cirúrgicas durante a evolução (12-18). Outros fatores que devem ser considerados são a dificuldade de anticoagulação dos pacientes em nosso meio e a calcificação precoce das próteses biológicas nesta faixa etária.

$\mathrm{Na}$ regurgitação mitral o tratamento cirúrgico tem sido prorrogado até o surgimento de algum grau de disfunção ventricular ou desenvolvimento de sintomas significantes (12). No entanto, o sucesso do tratamento cirúrgico sugere que estes parâmetros devam ser reexaminados $(9,10,12,13)$. UVA et al. ${ }^{(12)}$ advogam que a plastia mitral deve ser indicada

GRÁFICO 1

CURVA DE SOBREVIDA ACTUARIAL.

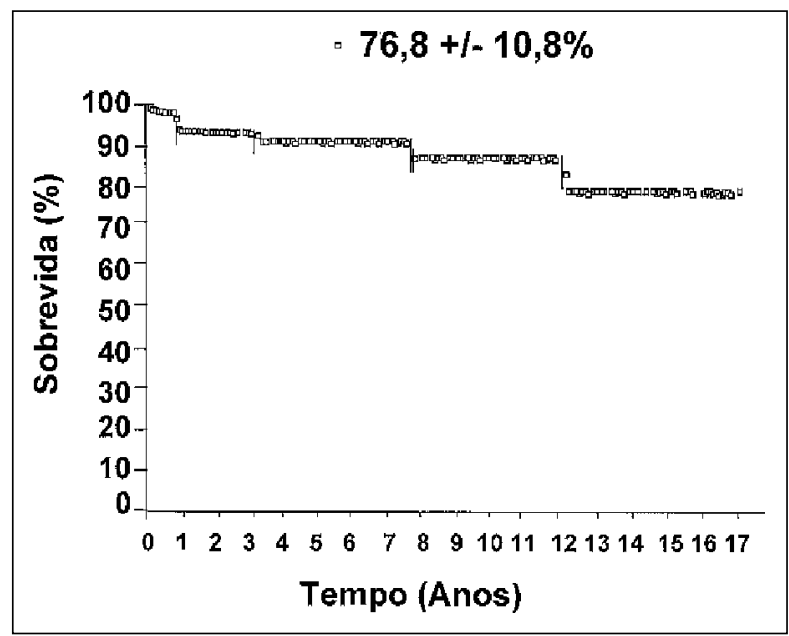


Pomerantzeff P M A, Brandão C M A, Faber C N, Fonseca M H, Puig L B, Grinberg M, Cardoso L F, Tarasoutchi F, Stolf N A G, Verginelli G, Jatene A D - Plástica da valva mitral: resultados aos 17 anos de experiência. $\quad$ Rev Bras Cir Cardiovasc 1999; 14 (3): 185-90.

GRÁFICO 2

CURVA DE SOBREVIDA ACTUARIAL LIVRE DE ENDOCARDITE.

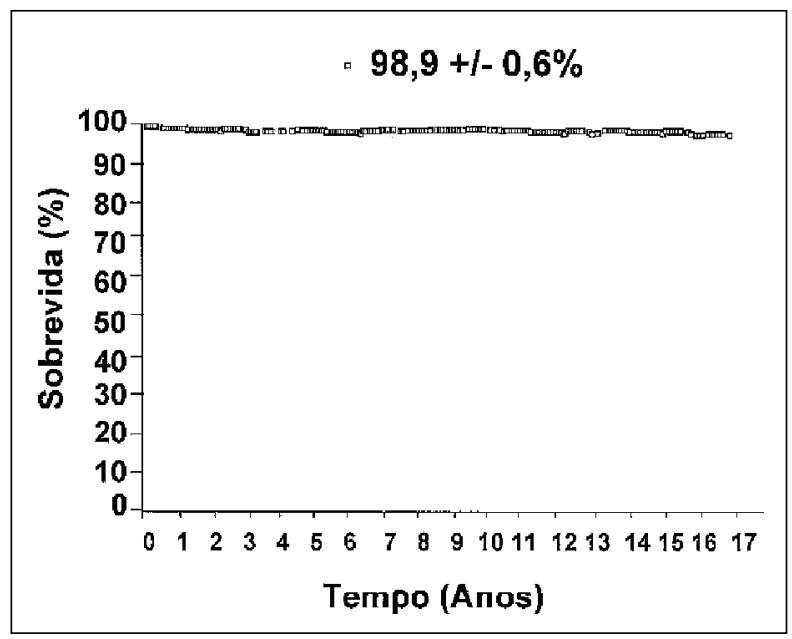

precocemente com base na gravidade da regurgitação e grau de comprometimento valvar que possibilite o tratamento conservador apesar da escassez ou ausência de sintomas. Em nosso estudo a maioria dos pacientes foi operada numa fase avançada de comprometimento valvar, daí o grande número de doentes em classe funcional IV. Este fato é devido em parte à dificuldade do acompanhamento clínico dos pacientes em nosso meio, uma vez que muitos já possuem importante grau de disfunção valvar no momento do diagnóstico.

A operação da valva mitral tem como objetivo a correção das alterações hemodinâmicas que causam a deterioração da função ventricular. Vários são os autores (19-21) que destacam a importância da manutenção da continuidade das cúspides, cordas tendíneas, músculos papilares para a preservação da função ventricular esquerda. BONCHEK et al. (21) concluíram em seu estudo que a plastia mitral

GRÁFICO 4

CURVA DE SOBREVIDA ACTUARIAL LIVRE DE REOPERAÇÃO.

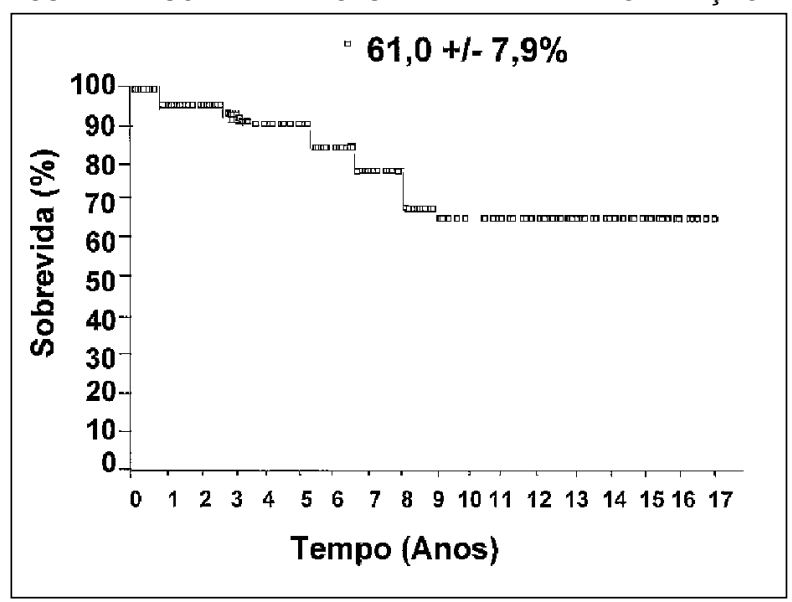

GRÁFICO 3 CURVADESOBREVIDAACTUARIAL LIVREDETROMBOEMBOLISMO.

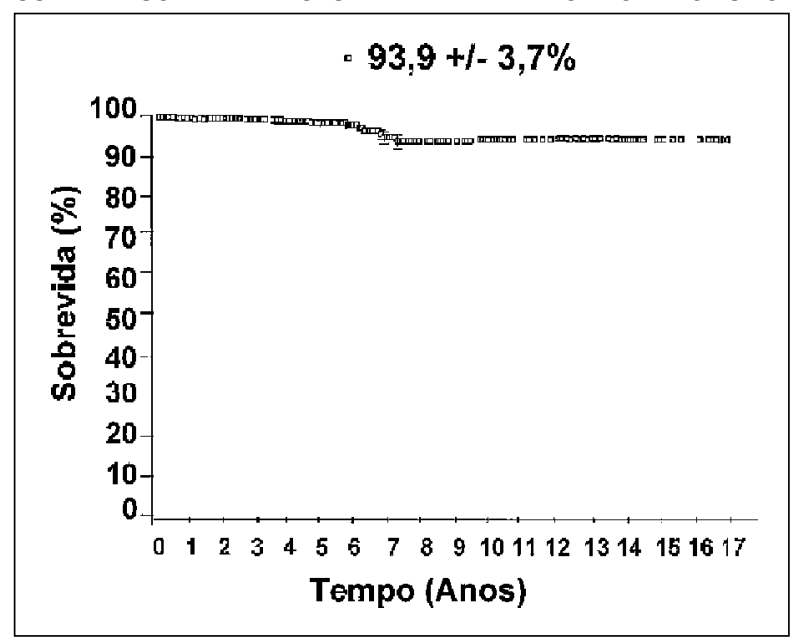

preserva o efeito de tração exercido pelas cordas tendíneas sobre a parede ventricular, prevenindo a dilatação ventricular esquerda no pós-operatório.

A reconstrução valvar exige do cirurgião um perfeito conhecimento da anatomia e da multiplicidade de técnicas existentes. Além disso, é importante a excelente visibilização da valva, especialmente do plano subvalvar, na tentativa de se conseguir uma boa avaliação das cordas tendíneas e musculatura papilar. Caso necessário procede-se ao encurtamento destas estruturas, na tentativa de uma boa coaptação das cúspides $(5,14)$. Em nosso Serviço temos preferido, em crianças, realizar o encurtamento do músculo papilar através da ressecção em cunha transversal, possibilitando um encurtamento mais homogêneo das cordas tendíneas.

A escolha da técnica a ser empregada para anuloplastia deve levar em consideração a altera-

GRÁFICO 5

CURVA DE SOBREVIDA ACTUARIAL LIVRE DE HEMÓLISE.

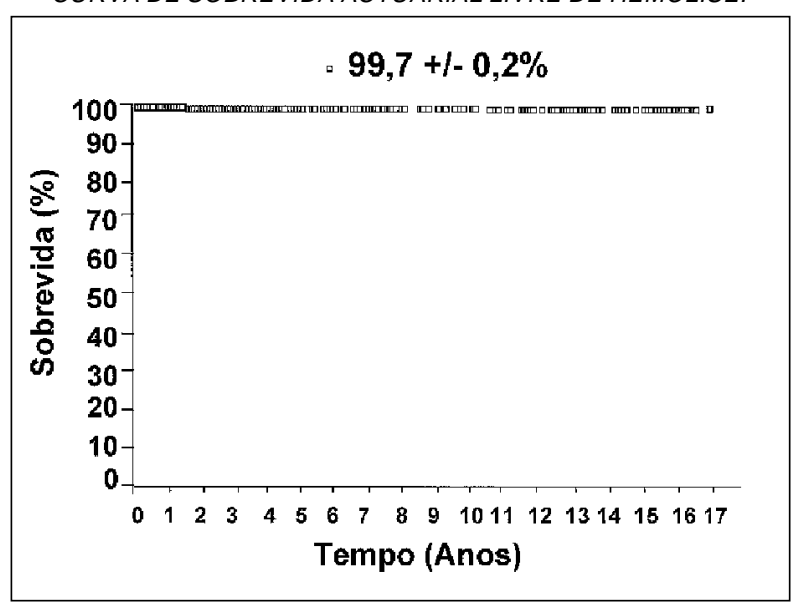


Pomerantzeff P M A, Brandão C M A, Faber C N, Fonseca M H, Puig L B, Grinberg M, Cardoso L F, Tarasoutchi F, Stolf N A G, Verginelli G, Jatene A D - Plástica da valva mitral: resultados aos 17 anos de experiência. $\quad$ Rev Bras Cir Cardiovasc 1999; 14 (3): 185-90.

ção valvar. Habitualmente, a dilatação do orifício mitral acomete apenas os dois terços posteriores do anel, o que corresponde a área da cúspide posterior. Vários anéis, rígidos ou flexíveis (22-24), foram descritos para remodelação da valva mitral. LOOP et al. (14) dão preferência à utilização do anel de Carpentier. Apesar de muito utilizados, os anéis rígidos podem apresentar alguns problemas tais como movimento sistólico anterior em $4 \%$ a $10 \%$ dos casos, causando a obstrução da via de saída de ventrículo esquerdo ${ }^{(25)}$. Em nosso Serviço temos utilizado esta técnica preferencialmente nas grandes dilatações do ventrículo esquerdo; nas demais situações, onde é necessário a redução do anel, utilizamos uma tira de pericárdio bovino.

ALVAREZ et al. (25) concluem em seu estudo que a anuloplastia com anel rígido não é pré-requisito absoluto para maior durabilidade da plastia na maioria dos pacientes com doença degenerativa da valva mitral. Acreditamos que o anel ideal ainda não foi idealizado. Este deveria ser flexível, ajustável e suficientemente extensível para permitir a dinâmica normal do anel mitral, sem perder a sua capacidade de contenção do anel posterior.

Nos casos de rotura de cordas tendíneas da cúspide posterior em pacientes portadores de degeneração mixomatosa, temos realizado a ressecção quadrangular da cúspide posterior e sutura borda a borda obtendo resultados similares aos da literatura $(26,27)$. Para estes pacientes, a partir de abril de 1994 temos utilizado a técnica do "Duplo Teflon", com bons resultados (28).

No que diz respeito à mortalidade hospitalar, BERNAL et al. (29), estudando 62 pacientes portadores de insuficiência mitral reumática submetidos à plástica da valva mitral durante um período de 20 anos, mostram uma taxa de $6,5 \%$, enquanto ALVAREZ et al. ${ }^{(25)}$, estudando 155 pacientes submetidos à plástica valvar por degeneração mixomatosa durante um período de 15 anos, apresentam uma taxa de $3,9 \%$. Nosso estudo é composto principalmente de pacientes portadores de degeneração mixomatosa e doença reumática, sendo a taxa de mortalidade hospitalar de $3,1 \%$, o que está de acordo com a literatura $(25,27-29)$.

Quanto à sobrevida actuarial, DELOCHE et al. (30) mostram uma taxa de $72,4 \%$ em 15 anos, o que corrobora os bons resultados por nós obtidos com uma sobrevida atuarial de $76,8 \%$ em 17 anos. Em relação aos porcentuais de sobrevida livre de tromboembolismo, reoperação e endocardite, nossos dados são comparáveis aos de outros autores $(13,21,27,30,31)$

Em conclusão, a plastia da valva mitral deve sempre ser considerada no tratamento cirúrgico da valva mitral por permitir melhora significativa do estado clínico dos pacientes, baixa mortalidade hospitalar e alta taxa de sobrevida na evolução tardia.

RBCCV 44205-459

Pomerantzeff P M A, Brandão C M A, Faber C N, Fonseca M H, Puig L B, Grinberg M, Cardoso L F, Tarasoutchi F, Stolf N A G, Verginelli G, Jatene A D - Mitral valve repair: seventeen years experience. Rev Bras Cir Cardiovasc 1999; 14 (3): 185-90.

ABSTRACT: From March 1980 to December 1997, 545 patients underwent 560 mitral valve repairs at the Heart Institute of HCFMUSP. Ages ranged from 3 months to 86 years with mean age of 42.2 and standard deviation of 21.4 years. Two hundred and seventy four (50.3\%) were male. Ethiology was rheumatic disease in 234 (42.9\%) patients. The techniques used were: quadrangular resection of posterior leaflet in $204(36.5 \%)$ patients, annuloplasty with posterior sling in 139 (24.5\%), Carpentier ring annuloplasty in $102(18.2 \%)$, segmentary posterior annuloplasty in $37(6.6 \%)$ and others. Associated procedures were performed in 267 $(35.6 \%)$ patients with the most frequent tricuspid valve repair in $95(17 \%)$ patients. Immediate mortality was $3.7 \%$ (21 patients). The linearizated rates of reoperation, thromboembolism, endocarditis and hemolysis, were respectively $2.9 \%, 0.6 \%, 0.3 \%$ and $0.1 \%$ patient/ year. The actuarial survival rate at 17 years was $76.8 \pm 10.8 \%$ and the actuarial freedom from endocarditis, thromboembolism, reoperation and hemolisis at 17 years was 98.9 $\pm 0.6 \%, 93.9 \pm 3.7 \%, 61.0 \pm 7.9 \%$ and $99.7 \pm 0.2 \%$, respectively. We conclude that patients submitted to mitral valve repair presented satisfactory evolution.

DESCRIPTORS: Mitral valve, surgery. Heart valve disease, surgery. 
Pomerantzeff P M A, Brandão C M A, Faber C N, Fonseca M H, Puig L B, Grinberg M, Cardoso L F, Tarasoutchi F, Stolf N A G, Verginelli G, Jatene A D - Plástica da valva mitral: resultados aos 17 anos de experiência. $\quad$ Rev Bras Cir Cardiovasc 1999; 14 (3): 185-90.

\section{REFERÊNCIAS BIBLIOGRÁFICAS}

1 Sundt T M 3rd, Khaghani A, Yacoub M H - Alternative technique for assessment and repair of the mitral valve. Ann Thorac Surg 1996; 61: 1552-4.

2 Stewart W J, Currie P J, Salcedo E E et al. - Evaluation of mitral leaflet motion by echocardiography and jet direction by Doppler color flow mapping to determine the mechanisms of mitral regurgitation. J Am Coll Cardiol 1992; 20: 1353- 61.

3 Carpentier A, Relland J, Deloche A et al. - Conservative management of the prolapsed mitral valve. Ann Thorac Surg 1978; 26: 294-302.

4 Pomerantzeff P M A, Brandão C M A, Monteiro A C M et al. - Plástica da valva mitral: resultados tardios de doze anos de experiência e evolução das técnicas. Rev Bras Cir Cardiovasc 1994; 9: 22-8.

5 Shore D F, Wong $\mathrm{P}$, Paneth $\mathrm{M}$ - Valve repair versus replacement in the surgical management of ruptured chordae: a post-operative echocardiographic assessment of mitral valve function. $J$ Cardiovasc Surg (Torino) 1982; 23: 378-82.

Anderson R P, Boncheck L I, Grunkemeier G L, Lambert L E, Starr $A$ - The analysis and presentation of surgical results by actuarial methods. J Surg Res 1974; 16: 224-30.

Grunkemeier G L - Statistical analysis of prosthetic valve series. In: Heart valve replacement and future trends in cardiac surgery. Mount Kisco, New York: Futura Publishing Company, 1987: 11-26.

Lillehei C W, Gott V L, Bonnabeau R C Jr - Surgical correction of pure mitral insufficiency by annuloplasty under direct vision. Lancet 1957; 77: 406-9.

9 Espada R \& Westaby S - New developments in mitral valve repair. Curr Opin Cardiol 1998; 13: 80-4.

10 Gillinov A M, Cosgrove D M, Blackstone E H et al. Durability of mitral valve repair for degenerative disease. J Thorac Cardiovasc Surg 1998; 116: 734-43.

11 Yacoub M, Halim M, Radley-Smith R, McKay R, Nijveld $A$, Towers $M$ - Surgical treatment of mitral regurgitation caused by floppy valves: repair versus replacement. Circulation 1981; 64(2Pt2): II210-6.

12 Uva M S, Dreyfus G, Rescigno G et al. - Surgical treatment of asymptomatic and mildly symptomatic mitral regurgitation. J Thorac Cardiovasc Surg 1996; 112: $1240-9$

Lee E M, Shapiro L M, Wells F C - Importance of subvalvular preservation and early operation in mitral valve surgery. Circulation 1996; 94: 2117-23.

Loop F D, Cosgrove D M, Stewart W J - Mitral valve repair for mitral insufficiency. Eur Heart $J$ 1991; 12(Suppl B): 30-3.

Pomerantzeff P M A, Brandão C M A, Faber C N et al. - Plástica da valva mitral em portadores de febre reumática. Rev Bras Cir Cardiovasc 1998; 13: 211-5.
16 David T E - The appropriateness of mitral valve repair for rheumatic mitral valve disease. J Heart Valve Dis 1997; 6: 373-4

17 Kumar A S, Rao P N, Saxena A - Mitral valve reconstruction: eight years'experience in 531 patients. J Heart Valve Dis 1997; 6: 591-3.

18 Cotrufo M, Vitale N, Cafarella G, De Feo M - Valve repair in rheumatic mitral valve disease: is it always worth trying? J Heart Valve Dis 1997; 6: 370-2.

19 Westaby S - Preservation of left ventricular function in mitral valve surgery. Heart 1996; 75: 326-9.

20 Salter D R, Pellom G L, Murphy C E et al. - Pappilaryannular continuity and left ventricular systolic function after mitral valve replacement. Circulation 1986; 74(3Pt2): I121-9

21 Bonchek L I, Olinger G N, Siegel R, Tresch D D, Keelan $\mathrm{M} \mathrm{H} \mathrm{Jr}$ - Left ventricular performance after mitral reconstruction for mitral regurgitation. J Thorac Cardiovasc Surg 1984; 88: 122-7.

22 Carvalho RG, Giublin PR, Lopes LR, Mulinari L, Loures DRR - Plástica da valva mitral com emprego do anel de Gregori-Braile: análise de 66 pacientes. Rev Bras Cir Cardiovasc 1998, 13: 295-316.

23 Gregori F Jr, Silva SS, Hayashi SS, Aquino W, Cordeiro C, Silva LR - Mitral valvuloplasty with a new prosthetic ring: analysis of the first 105 cases. Eur J Cardiothorac Surg 1994; 8: 168-72.

24 Carpentier A - La valvuloplastie reconstitutive: une nouvelle technique de valvuloplastie mitrale. Presse Med 1969; 77: 251-3.

25 Alvarez J M, Deal C W, Loveridge K et al. - Repairing the degenerative mitral valve: ten-to fifteen-year followup. J Thorac Cardiovasc Surg 1996; 112: 238-47.

26 Orszulak T A, Schaff H V, Danielson G K et al. - Mitral regurgitation due to ruptured chordae tendineae: early and late results of valve repair. $J$ Thorac Cardiovasc Surg 1985; 89: 491-8.

27 Perier P, Stumpf J, Gotz C et al. - Valve repair for mitral regurgitation caused by isolated prolapse of the posterior leaflet. Ann Thorac Surg 1997; 64: 445-50.

28 Pomerantzeff P M A, Brandão C M A, Rossi E G et al. - Quadrangular resection without ring annuloplasty in mitral valve repair. Cardiovasc Eng 1997; 2: 271-3.

29 Bernal J M, Rabasa J M, Olalla J J, Carrion M F, Alonso A, Revuelta J M - Repair of chordae tendineae for rheumatic mitral valve disease: a twenty-year experience. J Thorac Cardiovasc Surg 1996; 111: 211-7.

30 Deloche A, Jebara V A, Relland J Y et al. - Valve repair with Carpentier techniques: the second decade. $J$ Thorac Cardiovasc Surg 1990; 99: 990-1002.

31 Braile DM, Ardito RV, Pinto GH et al. - Plástica mitral. Rev Bras Cir Cardiovasc 1990; 5: 86-98. 